\title{
非预期的重排反应及苯并噁唑类化合物的合成
}

\author{
王凯嫙王兰芝* \\ (河北师范大学化学与材料科学学院 石家庄 050024)
}

\begin{abstract}
摘要 报道了一类酸促进的非预期的重排反应，合成了 6 个 2-芳基-3-(3-氧代丁烯基)-苯并噁唑化合物 $\mathbf{6 a} \sim \mathbf{6 f}$; 提出了 各步反应的反应机理, 采用密度泛函理论(DFT)方法, 从原子布居电荷和分子能量两方面对机理进行理论分析. 研究结 果表明, 理论和实验一致说明本文中的反应机理的正确. 重排反应机理的提出, 为进一步研究该类反应提供了依据, 为氮杂环类化合物的合成提供了方法参考.
\end{abstract}

关键词＼cjkstart重排反应; 苯并噁唑衍生物; 合成; 反应机理; 密度泛函理论(DFT)

\section{Unexpected Rearrangement Reaction and Synthesis of Benzoxazoles}

\author{
Wang, Kaixuan Wang, Lanzhi* \\ (College of Chemistry and Material Science, Hebei Normal University, Shijiazhuang 050024)
}

\begin{abstract}
Novel series of rearrangement reactions were herein reported that enable access to a variety of unique 2-aryl3-(3'-oxobutenyl)-benzoxazole compounds $\mathbf{6 a} \sim \mathbf{6}$ from 2-aminophenol, aromatic aldehyde and 3-butyn-2-one as materials by nucleophilic conjugate addition, dehydration and rearrangement reactions and intramolecular cyclization in the presence of a catalytic amount of $\mathrm{CH}_{3} \mathrm{COOH}$ in $\mathrm{CH}_{2} \mathrm{Cl}_{2}$ at ambient temperature. On the basis of products and intermediate products, a series of possible mechanism was presented and theoretically verified by density functional theory (DFT) method at B3LYP/6-31G (d,p) level from both molecular energy and atomic charge in Gaussian 03 package. The results show that the theory and experiment consistently explain the rationality of the reaction mechanism. The mechanism of rearrangement provides a basis for further study of this type of reaction. The advantage of this method is that a novel structure of benzoxazole derivative was synthesized successfully via a series of rearrangement reactions. Therefore, this method can be used as an attractive strategy for practical synthesis of nitrogen heterocyclic compounds.
\end{abstract}

Keywords rearrangement reaction; benzoxazole; synthesis; reaction mechanism; density functional theory (DFT)

氮杂环化合物种类繁多, 是有机化学中重要的组成 部分, 因部分杂环片段是重要的药效团, 将其引入到不 同的母体中可以改善或改变母体化合物的生物活性 ${ }^{[1]}$. 研究表明氮杂环化合物具有多种生物活性 ${ }^{[2 \sim 5]}$, 例如显 著的抑菌活性 ${ }^{[6]}$, 还具有抗病毒 ${ }^{[7,8]}$ 、抗癌 ${ }^{[9,10]}$ 、抗炎 ${ }^{[11]}$ 、 镇痛 ${ }^{[2,13]}$ 等作用. 其各类衍生物在农用化学品、天然产 物、功能材料 ${ }^{[14]}$ 和药物活性分子中应用也十分广泛 ${ }^{[15]}$, 例如以氮杂环化合物为起始原料可以合成苂光增白 剂 ${ }^{[16]}$, 作为配体和金属组成配合物 ${ }^{[17]}$ 等; 在医药上, 大 量的氮杂环化合物已经作为药物 ${ }^{[18]}$ 或候选物应用于临 床来治疗各种类型的疾病, 具有广阔的发展前景和应用
价值. 因此, 含氮杂环化合物的分子设计、合成及其生 物活性的研究已成为创造新农药的热点之一, 受到了化 学工作者和药理研究者的关注 ${ }^{[19,20]}$.

密度泛函理论(Density functional theory, DFT) 是一 种研究多电子体系电子结构的量子力学方法, 越来越多 的研究者将密度泛函中的 B3LYP 方法应用于量子化学 计算来解释反应中的机理 ${ }^{[21]}$.

本课题组一直致力于苯并氮杂环化合物, 特别是 1,5-苯并氮杂草类化合物的绿色合成及生物活性研 究 ${ }^{[22]}$, 前期我们已成功发展了一种新颖的合成 2-芳基3-乙酰基-1,5-苯并二氮杂草化合物的方法 ${ }^{[23]}$ (图 1a).

\footnotetext{
* Corresponding author. E-mail: wanglanzhi @ 126.com

Received September 28, 2018; revised November 14, 2018; published online January 9, 2019.

Project supported by the National Natural Science Foundation of China (Nos. 21776060, 21276064), and the Natural Science Foundation of Hebei Province (No. B2016205165).

国家自然科学基金(Nos. 21776060, 21276064)、河北省自然科学基金(No. B2016205165)资助项目.
} 


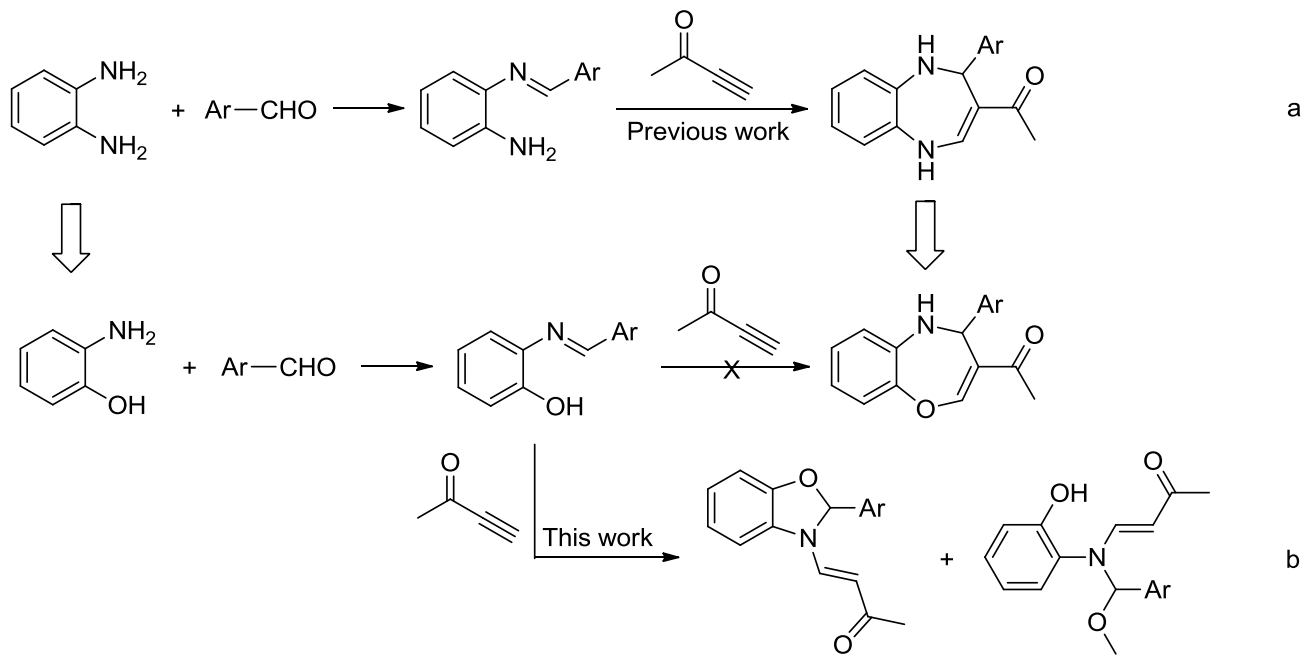

图 1 前期结果与研究计划

Figure 1 Preliminary results and research plan

鉴于此，本文也采用 2-氨基苯酚、芳香醛和 3-丁炔-2酮为起始原料进行系列分步反应, 以期合成 2-芳基-3乙酰基-1,5-苯并氧氮杂草化合物, 但是实验结果并没有 得到预期 1,5-苯并氧氮杂草化合物, 而是得到了另外两 种非预期产物苯并噁唑衍生物和烯胺酮类化合物 (图 $1 b$ ). 本文对该反应过程进行了分析，提出了各步反应 机理. 分析其主要原因是发生了一种特殊的重排反应, 得到了 2-芳基-3-(3-氧代丁烯基)-苯并噁唑化合物. 针对 其重排反应原因和重排动力, 采用密度泛函理论(DFT) 方法进行了理论分析.

\section{1 结果与讨论}

\section{1 系列反应及苯并噁唑衍生物的合成}

首先, 以乙醇为溶剂, 邻氨基苯酚 (1) 和芳香醛 $(\mathbf{2 a} \sim 2 \mathrm{f})$ 通过亲核加成、脱水得到亚胺类化合物 $3 \mathrm{a} \sim 3 \mathbf{f}$. 然后, 以三乙胺为催化剂, 3-丁炔-2-酮与亚胺类化合物 3a 3f 发生迈克尔加成反应得到中间体化合物 $\mathbf{5 a} \sim \mathbf{5 f}$. 中间体 5 以二氯甲烷为溶剂、冰醋酸为催化剂, 室温条 件下 $4 \sim 5 \mathrm{~h}$ 反应完全, 析出黄色固体, 得到目标化合物 2-芳基-3-(3-氧代丁烯基)-苯并噁唑化合物 $\mathbf{6 a} \sim \mathbf{6 f}$, 产率 $78 \%$ 92\%. 当中间体 5 以甲醇为溶剂、氯化铈为催化 剂时, 室温下反应 $4 \sim 5 \mathrm{~h}$, 析出淡黄色固体, 得到烯胺 酮类化合物 7, 反应过程如 Scheme 1 所示.

\section{2 晶体结构}

我们得到了产物 $\mathbf{6 c}$ 和 7 的单晶, 利用 $X$ 射线单晶 衍射法测定了两类化合物的单晶结构, 结果如图 2 和 3 所示. 数据存于英国剑桥数据中心, 化合物 $\mathbf{6 c}$ 的 CCDC 号为 1590453 , 化合物 7 的 CCDC 号为 1868579 .
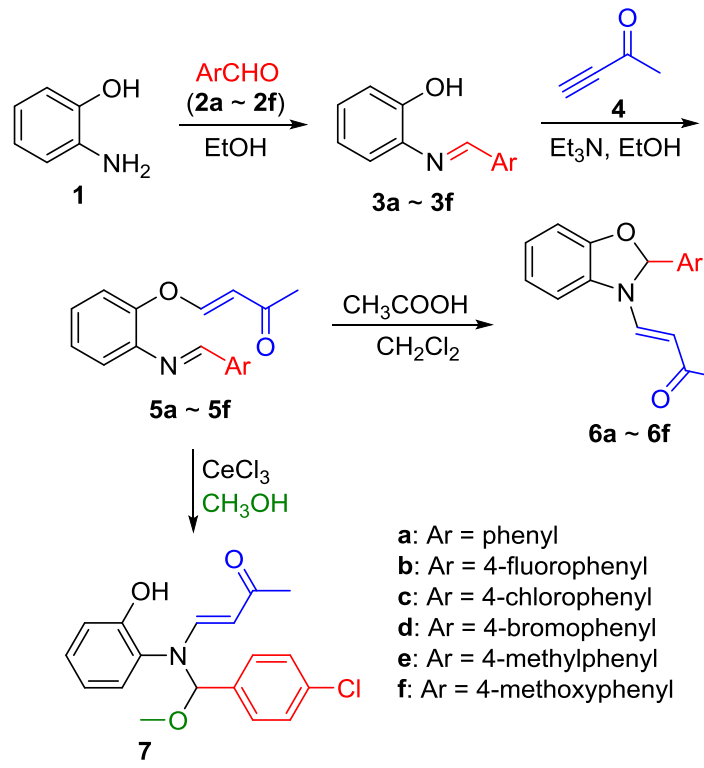

a: $\mathrm{Ar}=$ phenyl

b: $A r=4$-fluorophenyl

c: $\mathrm{Ar}=4$-chlorophenyl

d: $\mathrm{Ar}=4$-bromophenyl

e: $A r=4-m e t h y l p h e n y l$

f: $\mathrm{Ar}=4$-methoxyphenyl

图式 1 苯并噁坐衍生物的合成路径

Scheme 1 Synthetic route of benzoxazole

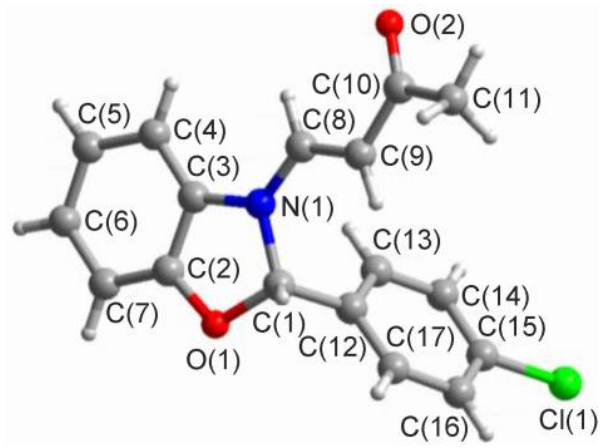

图 2 化合物 $\mathbf{6 c}$ 的单晶结构

Figure 2 Crystal structure of compound $\mathbf{6 c}$ 


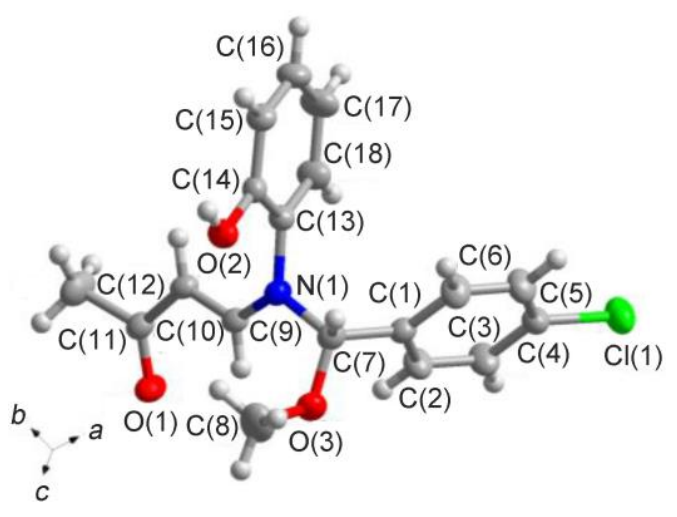

图 3 化合物 7 的单晶结构

Figure 3 Crystal structure of compound 7

\section{3 反应机理}

根据反应过程及各步反应的中间体, 提出了可能 的反应机理, 如 Scheme 2 所示.

首先, 2-氨基苯酚 $\mathbf{1}$ 中的氨基进攻芳香醛 $\mathbf{2}$ 的醛基, 发生亲核加成, 脱水反应生成含有亚胺结构的中间体化 合物 3 , 然后化合物 3 中的羟基在三乙胺的作用下失去 氢形成中间体 $\mathbf{A}, \mathbf{A}$ 中的酚氧负离子与 3-丁炔-2-酮发生 共轭加成反应生成中间体 5. 按路线 I: 在质子酸作用下 化合物 5 中的亚胺双键发生极化(活化), 带负电荷的氧 原子进攻活化后的 $\mathrm{C}=\mathrm{N}$, 发生亲核加成、分子内环化 反应生成活性中间体 $\mathbf{B}$. 在质子酸作用下, 中间体 $\mathbf{B}$ 中 氮负离子发生分子内的迈克尔加成形成中间体 $\mathbf{C}, \mathbf{C}$ 发 生消除反应(碳氧键断裂), 再经烯醇式与酮式互变生成 终产物 2-芳基-3-(3-氧代丁烯基)-苯并噁唑化合物 6. 按 路线 II: 在路易斯酸 $\mathrm{CeCl}_{3}$ 作用下, 中间体 $\mathbf{5}$ 中 $\mathrm{C}=\mathrm{N}$ 同
样发生极化(活化)，溶剂甲醇中氧原子的亲核性比醚键 中的氧原子强，所以甲醇中的氧作为亲核中心进攻活化 后的 $\mathrm{C}=\mathrm{N}$, 发生亲核加成反应得到活性中间体 $\mathbf{D}, \mathbf{D}$ 发 生分子内迈克尔加成形成 $\mathbf{E}, \mathbf{E}$ 发生消除反应(碳氧键异 裂), 烯醇转酮式, 最终得到产物 7.

由上述机理 Scheme 2 可知, 由中间体 $\mathbf{5}$ 生成最终产 物的实质其实就是两步反应: 一步是氧原子对活化后的 亚胺 $(C=N)$ 的亲核加成, 在这一步有一对竞争反应, 就 是醚键中的氧和甲醇中的氧均具有亲核性, 但甲醇中氧 的亲核性较高, 当甲醇存在时, 按路径 II 进行; 当反应 体系内没有甲醇时，醚键中亲核性的氧与亚胺双键发生 亲核加成反应, 按路径 I 进行. 另一步反应是氮负离子 的加成一消除(碳氧键异裂)及烯醇变酮式过程, 经过这 样特殊的重排反应得到了具有图中结构的产物. 产物 7 的生成也进一步验证了化合物 6 的形成机理.

\section{4 理论分析}

\subsection{1 布居电荷角度分析形成机理}

在机理 Scheme 2 中, 中间产物 $\mathbf{3}$ 和 $\mathbf{5}$ 的结构均经 ${ }^{1} \mathrm{H}$ NMR 进行了确认, 发现化合物 5 是一个非常重要的中 间产物, 其结构与电荷分布均影响到下一步反应和终产 物的生成, 因此本文采用 Gaussian 03 程序中密度泛函 理论(DFT)方法在 B3LYP/6-31G(d,p)基组水平上 ${ }^{[24]}$, 对 化合物 5 进行了几何优化和量化计算, 得到了化合物 $\mathbf{5}$ 的各原子布居电荷, 具体见表 1 所示. 通过表 1 分析, 以 期对机理进行理论验证. 表 1 中列出了根据 DFT 方法计 算出的化合物 5 中的 $\mathrm{O}(7), \mathrm{N}(8), \mathrm{C}(14)$ 原子的布居电荷.

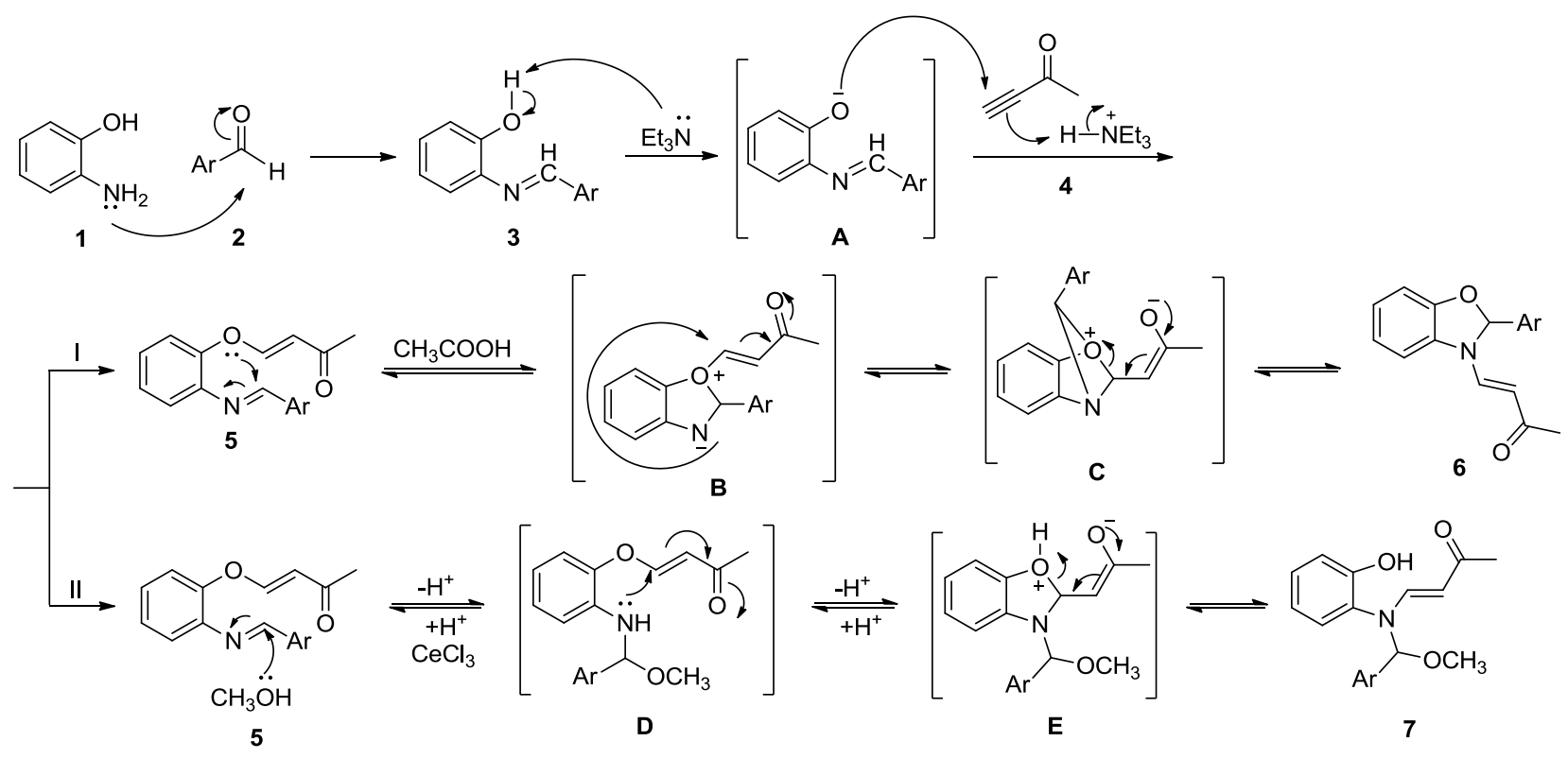

图式 2 可能的反应机理

Scheme 2 A possible reaction mechanism 
表 1 化合物 5 中 $\mathrm{O}(7), \mathrm{N}(8)$ 和 $\mathrm{C}(14)$ 的电荷密度(a.u.) Table 1 Charge density (a.u.) of $\mathrm{O}(7), \mathrm{N}(8)$ and $\mathrm{C}(14)$ in compound 5

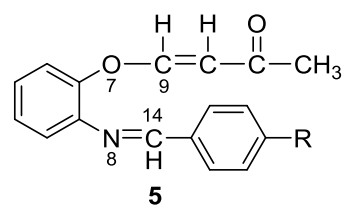

\begin{tabular}{ccccc}
\hline Compd. & $\mathrm{R}$ & $\mathrm{O}(7)$ & $\mathrm{N}(8)$ & $\mathrm{C}(14)$ \\
\hline $\mathbf{5 a}$ & $\mathrm{H}$ & -0.4574 & -0.4296 & 0.1166 \\
$\mathbf{5 b}$ & $\mathrm{F}$ & -0.4750 & -0.4366 & 0.1142 \\
$\mathbf{5 c}$ & $\mathrm{Cl}$ & -0.4757 & -0.4716 & 0.1213 \\
$\mathbf{5 d}$ & $\mathrm{Br}$ & -0.4964 & -0.4486 & 0.1209 \\
$\mathbf{5 f}$ & $\mathrm{OCH}_{3}$ & -0.4599 & -0.4421 & 0.1184 \\
\hline
\end{tabular}

由表 1 可知, 化合物 5 中 $\mathrm{C}(14)$ 带正电荷, $\mathrm{O}(7)$ 和 $N(8)$ 均带负电荷, 对比发现: $O(7)$ 上的电荷密度明显高 于 $N(8)$, 这使得 $\mathrm{O}(7)$ 比 $\mathrm{N}(8)$ 更具有亲核性, 所以在反应 中首先是亲核性的 $\mathrm{O}(7)$ 进攻带正电的 $\mathrm{C}(14)$ 发生亲核加 成形成 $\mathrm{C}-\mathrm{O}$ 键, 而不是 $\mathrm{N}(8)$ 进攻带正电的 $\mathrm{C}(9)$, 这一 结果也验证了本文提出的机理的合理性.

\subsection{2 能量角度分析产物的稳定性}

本文还运用 Gaussian 03 程序中密度泛函(DFT)方法 在 B3LYP/6-31G $(\mathrm{d}, \mathrm{p})$ 基组水平上对反应得到的产物苯 并噁唑化合物 6 及对应预期 1,5-苯并氧氮杂草化合物 8 进行了量化计算, 得到了各化合物的能量参数, 包括零 点能、热力学能、热力学焓及吉布斯自由能等, 计算结 果见表 2 所示. 从能量角度分析了产物的稳定性, 从热

表 2 化合物 6 和 8 的零点能 $\left(E_{\mathrm{zp}}\right)$ 和内能 $\left(E^{\ddagger}\right)$ 、热力学焓 $\left(H^{\ddagger}\right)$ 以及自由能 $\left(G^{\ddagger}\right)$

Table 2 Zero-point energies $\left(E_{\mathrm{zp}}\right)$, thermal energies $\left(E^{\ddagger}\right)$, thermal enthalpies $\left(H^{\ddagger}\right)$ and thermal Free energies $\left(G^{\ddagger}\right)$ of compounds 6 and 8

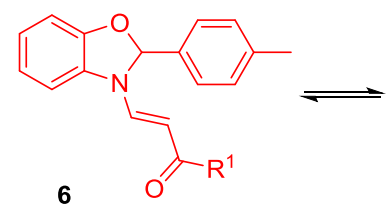

a $\sim$ f: $\mathrm{R}^{1}=\mathrm{H}, \mathrm{F}, \mathrm{Cl}, \mathrm{Br}, \mathrm{CH}_{3}, \mathrm{OCH}_{3}$

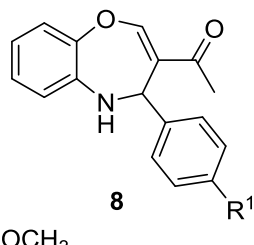

\begin{tabular}{ccccc}
\hline Compd. & $E_{\text {zp. }}{ }^{a}$ & $E^{\ddagger b}$ & $H^{\neq c}$ & $G^{\neq d}$ \\
\hline $\mathbf{6 a}$ & -861.7654 & -861.7483 & -861.7474 & -861.8126 \\
$\mathbf{8 a}$ & -861.7360 & -861.7194 & -861.7184 & -861.7811 \\
$\mathbf{6 b}$ & -960.9985 & -960.9814 & -960.9805 & -961.0454 \\
$\mathbf{8 b}$ & -960.9755 & -960.9580 & -960.9571 & -961.0217 \\
$\mathbf{6 c}$ & -1321.3687 & -1321.3504 & -1321.3495 & -1321.4182 \\
$\mathbf{8 c}$ & -1321.3399 & -1321.3219 & -1321.3209 & -1321.3881 \\
$\mathbf{6 d}$ & -3432.8712 & -3432.8525 & -3432.8516 & -3432.9232 \\
$\mathbf{8 d}$ & -3432.8491 & -3432.8309 & -3432.8299 & -3432.8977 \\
$\mathbf{6 f}$ & -976.2563 & -976.2366 & -976.2356 & -976.3079 \\
$\mathbf{8 f}$ & -976.2287 & -976.2093 & -976.2084 & -976.2779 \\
\hline${ }^{a}$ a.u. ${ }^{b}{ }^{b} 10^{3} \mathrm{~kJ} / \mathrm{mol}^{c}{ }^{c} 10^{3} \mathrm{~kJ} / \mathrm{mol}^{d}{ }^{d} 10^{3} \mathrm{~kJ} / \mathrm{mol}$. & &
\end{tabular}

力学角度得出稳定 (优势)产物为苯并噁唑化合物, 这与 实验事实相符.

由于本文的产物 2-芳基-3-(3-氧代丁烯基)-苯并惡 唑化合物 6 与预期的 1,5 -苯并氧氮杂草化合物 8 是同分 异构体，但是化合物的结构不同，计算得出各自能量参 数(零点能、热力学能、热力学焓及吉布斯自由能)也不 同. 由表 2 可知, 本文的产物苯并噁唑化合物 6 的能量 均低于相应的 1,5 -苯并氧氮杂草化合物 8 . 能量越低的 化合物结构越稳定，因此化合物 6 是优势结构，在反应 中容易生成. 这一研究结果与本文的实验事实相符.

对化合物 6c 进行理论计算优化后的分子结构如图 4a 所示, 通过与用 $\mathrm{X}$ 射线单晶衍射法测定的 $\mathbf{~ c ~}$ 的晶体 结构图 $4 \mathrm{~b}$ 对比发现, 两者的晶体结构一致. 表明采用 Gaussian 03 程序中密度泛函理论(DFT)方法在 B3LYP/ 6-31G $(\mathrm{d}, \mathrm{p})$ 基组水平上对化合物进行几何优化和量化计 算的方法是可靠的.
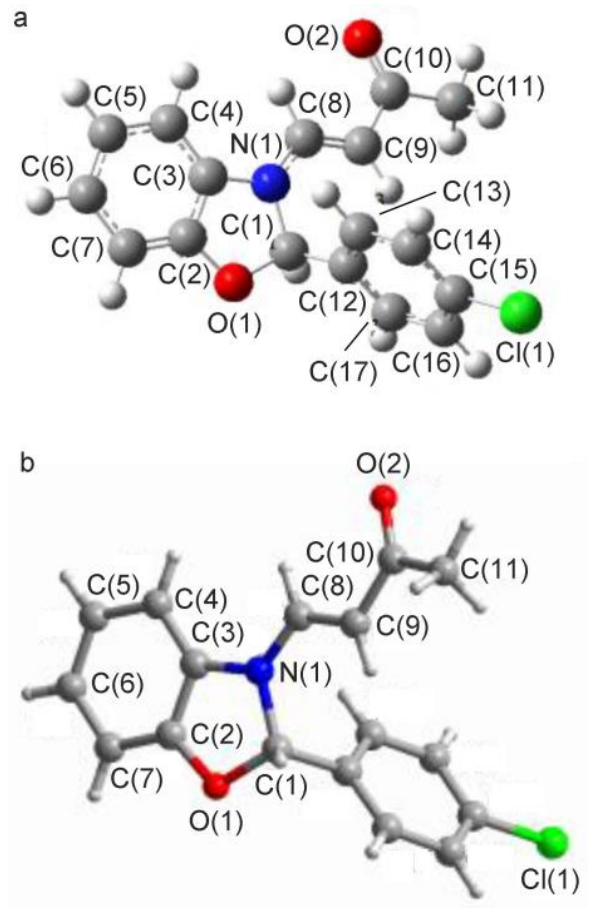

图 4 6c 优化的结构(a)与其单晶图(b)

Figure 4 Optimized structure (a) and crystal structure (b) of $\mathbf{6 c}$

\section{2 结论}

通过对系列反应考察，发现了一种在酸的作用下新 颖的重排反应，并得到 6 个未见文献报道的 2-芳基-3-(3氧代丁烯基)-苯并啞唑化合物，提出了系列反应详尽的 反应机理, 并由密度泛函理论(DFT)方法验证了该反应 机理, 反应机理的提出, 为进一步研究该类反应提供了 依据. 该重排反应条件温和, 产率较高, 且实验步骤操 作简单，为构建氮杂环类化合物提供了方法参考. 


\section{3 实验部分}

\section{1 仪器与试剂}

Thermo SCIENTIFIC 型红外光谱仪, KBr 压片法; 3200QTRAP 质谱仪; WIPM-NMR-400M 型核磁共振仪, TMS 为内标; Vario EL III 型元素分析仪. 2-氨基苯酚和 3-丁炔-2 酮均购自北京伊诺凯科技有限公司, 其它试剂 均为市售分析纯.

\section{2 实验方法}

3.2.1 中间体 4-[2-(4-卤代亚苄基氨基)苯氧基]-3-丁 烯-2-酮(5)的合成

向干燥的 $50 \mathrm{~mL}$ 圆底烧瓶中, 依次加入 $10 \mathrm{~mL}$ 无水 乙醇、邻氨基苯酚 $(2.0 \mathrm{mmol})$ 、取代苯甲醛 $(2.0 \mathrm{mmol})$ 室温下搅拌 $5 \mathrm{~min}$, 抽滤得到黄色固体 $\mathbf{3}$. 将 $\mathbf{3}$ 与 3 -丁 炔-2-酮按物质的量比 $1: 1$ 投入反应瓶, 加入 $0.02 \mathrm{~mL}$ 三乙胺, 磁力搅拌 $8 \mathrm{~min}$, 抽滤得到白色固体 $\mathbf{5}$.

4-[2-(4-氟代亚苄基氨基)苯氧基]-3-丁烯-2-酮(5b): 白色固体, 产率 95\%. m.p. 80 82 ${ }^{\circ} \mathrm{C} ;{ }^{1} \mathrm{H}$ NMR (400 MHz, DMSO- $\left.d_{6}\right) \delta: 8.65(\mathrm{~s}, 1 \mathrm{H}), 7.99 \sim 7.98(\mathrm{~m}, 2 \mathrm{H}), 7.94$ (d, $J=12.5 \mathrm{~Hz}, 1 \mathrm{H}), 7.37$ (t, $J=8.8 \mathrm{~Hz}, 2 \mathrm{H}), 7.32(\mathrm{~m}, 4 \mathrm{H})$, $5.67(\mathrm{~d}, J=12.5 \mathrm{~Hz}, 1 \mathrm{H}), 2.13(\mathrm{~s}, 3 \mathrm{H}) ;{ }^{13} \mathrm{C} \operatorname{NMR}(100$ MHz, DMSO- $\left.d_{6}\right) \quad \delta: 197.02,165.91,163.43,162.64$, $161.48,148.63,142.76,133.01,131.64,131.55,127.61$, $126.69,120.99,120.51,116.58,116.36,111.53,27.45$; IR (KBr) v: 1770, 1665, 1508, 1261, $934 \mathrm{~cm}^{-1}$; MS m/z: 284 $[\mathrm{M}+\mathrm{H}]^{+}$. Anal. calcd for $\mathrm{C}_{17} \mathrm{H}_{14} \mathrm{FNO}_{2}: \mathrm{C} 72.07, \mathrm{H} 4.98, \mathrm{~N}$ 4.94; found C 72.54, H 4.57, N 4.80.

4-[2-(4-氯代亚芐基氨基)苯氧基]-3-丁烯-2-酮 $(\mathbf{5 c})$ : 白色固体, 产率 98\%. m.p. $100 \sim 102{ }^{\circ} \mathrm{C} ;{ }^{1} \mathrm{H}$ NMR (400 MHz, DMSO- $\left.d_{6}\right) \delta: 8.66(\mathrm{~s}, 1 \mathrm{H}), 7.97 \sim 7.92(\mathrm{~m}, 2 \mathrm{H}), 7.96$ (d, $J=12.5 \mathrm{~Hz}, 1 \mathrm{H}), 7.37$ (t, $J=8.8 \mathrm{~Hz}, 2 \mathrm{H}), 7.32(\mathrm{~m}, 4 \mathrm{H})$, $5.64(\mathrm{~d}, J=12.5 \mathrm{~Hz}, 1 \mathrm{H}), 2.12(\mathrm{~s}, 3 \mathrm{H}) ;{ }^{13} \mathrm{C}$ NMR $(100$ MHz, DMSO- $\left.d_{6}\right) \quad \delta: 197.06,162.66,161.57,148.70$, $142.57,136.94,135.15,130.83,129.51,127.84,126.70$, 120.98, 120.53, 111.59, 27.45; IR (KBr) v: 1691, 1626, $1261,1046,752 \mathrm{~cm}^{-1} ; \mathrm{MS} \mathrm{m} / z: 300[\mathrm{M}+\mathrm{H}]^{+}$. Anal. calcd for $\mathrm{C}_{17} \mathrm{H}_{14} \mathrm{ClNO}_{2}$ : C 68.12, $\mathrm{H} 4.71, \mathrm{~N}$ 4.67; found $\mathrm{C} 68.36$, $\mathrm{H} 4.65, \mathrm{~N} 4.59$.

4-[2-(4-溴代亚芐基氨基)苯氧基]-3-丁烯-2-酮(5d), 白色固体, 产率 95\%. m.p. 104 106 ${ }^{\circ} \mathrm{C} ;{ }^{1} \mathrm{H}$ NMR (400 MHz, DMSO- $\left.d_{6}\right) \delta: 8.65(\mathrm{~s}, 1 \mathrm{H}), 7.94(\mathrm{~d}, J=12.5 \mathrm{~Hz}, 1 \mathrm{H})$, $7.86(\mathrm{~d}, J=8.3 \mathrm{~Hz}, 2 \mathrm{H}), 7.74(\mathrm{~d}, J=8.3 \mathrm{~Hz}, 2 \mathrm{H}), 7.37 \sim$ $7.30(\mathrm{~m}, 4 \mathrm{H}), 5.66(\mathrm{~d}, J=12.5 \mathrm{~Hz}, 1 \mathrm{H}), 2.13(\mathrm{~s}, 3 \mathrm{H}) ;{ }^{13} \mathrm{C}$ NMR $\left(100 \mathrm{MHz}\right.$, DMSO- $\left.d_{6}\right) \delta: 197.03,162.59,161.70$, $148.68,142.57,135.47,132.43,130.99,127.85,126.70$,
125.95, 120.96, 120.53, 112.48, 111.59, 27.45; IR (KBr) v: 1665, 1267, 1020, 948, $544 \mathrm{~cm}^{-1}$; MS m/z: $344[\mathrm{M}+\mathrm{H}]^{+}$. Anal. calcd for $\mathrm{C}_{17} \mathrm{H}_{14} \mathrm{BrNO}_{2}$ : C 59.32, H 4.10, $\mathrm{N}$ 4.07; found C 59.68, H 4.36, N 3.88.

3.2.2 2-苯基-3-(3-氧代丁烯基)-苯并噁唑(6)的合成 向干燥的 $50 \mathrm{~mL}$ 圆底烧瓶中, 依次加入 $5(2.0$ $\mathrm{mmol}$ )、 $10 \mathrm{~mL}$ 二氯甲烷, $0.06 \mathrm{~mL}$ 冰醋酸, 室温下反应 5 $\mathrm{h}$, 抽滤得到淡黄色固体 $\mathbf{6}$.

2-苯基-3-(3-氧代丁烯基)-苯并噁唑(6a)：淡黄色固 体, 产率 79\%. m.p. $136 \sim 138{ }^{\circ} \mathrm{C} ;{ }^{1} \mathrm{H}$ NMR $(400 \mathrm{MHz}$, DMSO- $\left.d_{6}\right) \delta: 7.99(\mathrm{~d}, J=13.9 \mathrm{~Hz}, 1 \mathrm{H}), 7.42 \sim 7.46(\mathrm{~m}$, $5 \mathrm{H}), 7.06(\mathrm{~s}, 1 \mathrm{H}), 6.86 \sim 7.45(\mathrm{~m}, 4 \mathrm{H}), 5.03(\mathrm{~d}, J=13.9$ $\mathrm{Hz}, 1 \mathrm{H}), 2.09$ (s, 3H); ${ }^{13} \mathrm{C}$ NMR (100 MHz, DMSO- $\left.d_{6}\right) \delta$ : 195.56, 149.78, 138.93, 136.03, 132.25, 130.76, 129.63, $126.92,123.90,122.20,109.51,109.40,105.56,94.89$, 26.98; IR (KBr) v: 1662, 1557, 1334, 962, $745 \mathrm{~cm}^{-1}$; MS $m / z: 266[\mathrm{M}+\mathrm{H}]^{+}$. Anal. calcd for $\mathrm{C}_{17} \mathrm{H}_{15} \mathrm{NO}_{2}: \mathrm{C} 76.96, \mathrm{H}$ 5.70, N 5.28; found C 76.76, H 5.58, N 5.46.

2-(4-氟苯基)-3-(3-氧代丁烯基)-苯并噁唑 $(\mathbf{6 b})$ : 淡 黄色固体, 产率 85\%. m.p. 112 114 ${ }^{\circ} \mathrm{C} ;{ }^{1} \mathrm{H}$ NMR (400 MHz, DMSO- $\left.d_{6}\right) \delta: 7.99(\mathrm{~d}, J=14.0 \mathrm{~Hz}, 1 \mathrm{H}), 7.28 \sim 7.52$ (m, 4H), $7.11(\mathrm{~s}, 1 \mathrm{H}), 6.88 \sim 7.54(\mathrm{~m}, 4 \mathrm{H}), 5.03(\mathrm{~d}, J=$ $14.0 \mathrm{~Hz}, 1 \mathrm{H}), 2.10$ (s, 3H); ${ }^{13} \mathrm{C}$ NMR (100 MHz, DMSO$\left.d_{6}\right) \delta: 195.24,164.43,162.47,149.67,138.80,132.57$, $132.22,129.46,129.39,123.85,122.21,116.71,116.53$, 109.63, 109.44, 105.74, 94.09, 27.08; IR (KBr) v: 1673, 1567, 1279, 964, $741 \mathrm{~cm}^{-1}$; MS m/z: $284[\mathrm{M}+\mathrm{H}]^{+}$. Anal. calcd for $\mathrm{C}_{17} \mathrm{H}_{14} \mathrm{FNO}_{2}$ : C 72.07, $\mathrm{H} 4.98, \mathrm{~N}$ 4.94; found $\mathrm{C}$ $72.50, \mathrm{H} 4.58, \mathrm{~N} 4.78$.

2-(4-氯苯基)-3-(3-氧代丁烯基)-苯并噁唑(6c)：淡 黄色固体, 产率 92\%. m.p. 130 132 ${ }^{\circ} \mathrm{C} ;{ }^{1} \mathrm{H}$ NMR (400 MHz, DMSO- $\left.d_{6}\right) \delta: 8.00(\mathrm{~d}, J=14.0 \mathrm{~Hz}, 1 \mathrm{H}), 7.48 \sim 7.55$ $(\mathrm{m}, 4 \mathrm{H}), 7.11(\mathrm{~s}, 1 \mathrm{H}), 6.88 \sim 7.50(\mathrm{~m}, 4 \mathrm{H}), 5.04(\mathrm{~d}, J=$ $14.0 \mathrm{~Hz}, 1 \mathrm{H}), 2.10$ (s, 3H); ${ }^{13} \mathrm{C}$ NMR (100 MHz, DMSO$\left.d_{6}\right) \delta: 195.23,149.69,138.76,135.09,129.73,128.94$, 123.87, 122.27, 109.66, 109.47, 105.78, 94.02, 27.12; IR (KBr) $v: 1668,1563,1276,974,752 \mathrm{~cm}^{-1}$; MS m/z: 300 $[\mathrm{M}+\mathrm{H}]^{+}$. Anal. calcd for $\mathrm{C}_{17} \mathrm{H}_{14} \mathrm{ClNO}_{2}: \mathrm{C} 68.12, \mathrm{H} 4.71$, N 4.67; found C 68.46, H 4.55, N 4.53.

2-(4-溴苯基)-3-(3-氧代丁烯基)-苯并噁唑(6d)：淡 黄色固体, 产率 88\%. m.p. 132 134 ${ }^{\circ} \mathrm{C} ;{ }^{1} \mathrm{H}$ NMR (400 MHz, DMSO- $\left.d_{6}\right) \delta: 7.99(\mathrm{~d}, J=14.0 \mathrm{~Hz}, 1 \mathrm{H}), 7.40 \sim 7.67$ (m, 4H), $7.09(\mathrm{~s}, 1 \mathrm{H}), 6.86 \sim 7.50(\mathrm{~m}, 4 \mathrm{H}), 5.04(\mathrm{~d}, J=$ $14.0 \mathrm{~Hz}, 1 \mathrm{H}), 2.09$ (s, 3H); ${ }^{13} \mathrm{C}$ NMR $(100 \mathrm{MHz}$, DMSO- $\left.d_{6}\right) \delta: 195.23,149.67,138.75,135.44,132.65$, 
$132.17,129.17,124.07,123.85,122.25,109.64,109.46$, 105.77, 94.06, 27.10; IR (KBr) v: 1669, 1563, 1275, 974, $738 \mathrm{~cm}^{-1}$; MS m/z: $344[\mathrm{M}+\mathrm{H}]^{+}$. Anal. calcd for $\mathrm{C}_{17} \mathrm{H}_{14} \mathrm{BrNO}_{2}$ : C 59.32, $\mathrm{H} 4.10, \mathrm{~N} 4.07$; found $\mathrm{C} 59.76, \mathrm{H}$ 4.33, N 3.91.

2-(4-甲基苯基)-3-(3-氧代丁烯基)-苯并啞唑(6e): 黄色固体, 产率 83\%. m.p. $138 \sim 140{ }^{\circ} \mathrm{C} ;{ }^{1} \mathrm{H}$ NMR (400 MHz, DMSO- $\left.d_{6}\right) \delta: 7.98(\mathrm{~d}, J=14.0 \mathrm{~Hz}, 1 \mathrm{H}), 7.24 \sim 7.35$ $(\mathrm{m}, 4 \mathrm{H}), 7.02(\mathrm{~s}, 1 \mathrm{H}), 6.85 \sim 7.51(\mathrm{~m}, 4 \mathrm{H}), 5.03(\mathrm{~d}, J=$ $13.9 \mathrm{~Hz}, 1 \mathrm{H}), 2.32$ (s, 3H), 2.09 (s, 3H); ${ }^{13} \mathrm{C}$ NMR $(100$ $\left.\mathrm{MHz}, \mathrm{DMSO}-d_{6}\right) \quad \delta: 195.19,149.84,140.34,138.80$, $133.30,132.34,130.10,126.92,123.74,122.05,109.47$, 109.32, 105.65, 94.90, 27.00, 21.33; IR (KBr) v: 1662, 1570, 1288, 981, $745 \mathrm{~cm}^{-1}$; MS m/z: $280[\mathrm{M}+\mathrm{H}]^{+}$. Anal. calcd for $\mathrm{C}_{18} \mathrm{H}_{17} \mathrm{NO}_{2}$ : C 77.40, $\mathrm{H}$ 6.13, N 5.01; found $\mathrm{C}$ 77.24, H 6.42, N 5.11.

2-(4-甲氧基苯基)-3-(3-氧代丁烯基)-苯并噁唑(6f): 黄色固体, 产率 78\%. m.p. 142 144 ${ }^{\circ} \mathrm{C} ;{ }^{1} \mathrm{H}$ NMR (400 MHz, DMSO- $\left.d_{6}\right) \delta: 7.94(\mathrm{~d}, J=14.0 \mathrm{~Hz}, 1 \mathrm{H}), 6.96 \sim 7.40$ $(\mathrm{m}, 4 \mathrm{H}), 7.01(\mathrm{~s}, 1 \mathrm{H}), 6.85 \sim 7.49(\mathrm{~m}, 4 \mathrm{H}), 5.04(\mathrm{~d}, J=$ $14.0 \mathrm{~Hz}, 1 \mathrm{H}), 3.77$ (s, 3H), 2.09 (s, 3H); ${ }^{13} \mathrm{C}$ NMR $(100$ MHz, DMSO- $\left.d_{6}\right) \delta: 195.20,161.02,149.81,138.79$, $132.33,128.53,128.24,123.73,122.00,114.90,109.44$, 109.30, 105.62, 94.83, 55.70, 27.00; IR (KBr) v: 1668, 1570, 1282, 967, $745 \mathrm{~cm}^{-1}$; MS m/z: $296[\mathrm{M}+\mathrm{H}]^{+}$. Anal. calcd for $\mathrm{C}_{18} \mathrm{H}_{17} \mathrm{NO}_{3}$ : C 73.20, $\mathrm{H}$ 5.80, N 4.74; found $\mathrm{C}$ 73.32, H 5.65, N 4.70.

\subsection{3 化合物 7 的合成}

向干燥的 $50 \mathrm{~mL}$ 圆底烧瓶中, 依次加入 5c (2.0 $\mathrm{mmol}) 、 10 \mathrm{~mL}$ 无水甲醇, 适量三氯化铈, 室温下反应 12 $\mathrm{h}$, 停止反应, 冰箱冷冻 $24 \mathrm{~h}$, 抽滤得到淡黄色固体 7 . 淡黄色固体, 产率 90\%. m.p. $138 \sim 140{ }^{\circ} \mathrm{C} ;{ }^{1} \mathrm{H}$ NMR $\left(400 \mathrm{MHz}, \mathrm{DMSO}-d_{6}\right) \delta: 9.46(\mathrm{~s}, 1 \mathrm{H}), 7.93(\mathrm{~s}, 1 \mathrm{H}), 6.77 \sim$ $7.31(\mathrm{~m}, 8 \mathrm{H}), 5.94(\mathrm{~s}, 1 \mathrm{H}), 4.70(\mathrm{~s}, 1 \mathrm{H}), 3.55(\mathrm{~s}, 3 \mathrm{H}), 1.98$ $(\mathrm{s}, 3 \mathrm{H}) ;{ }^{13} \mathrm{C}$ NMR (100 MHz, DMSO-d 6 ) $\delta$ : 194.97, 153.61, 136.99, 133.19, 129.67, 129.08, 128.22, 119.70, 117.007, 101.51, 94.78, 56.23, 27.74; IR (KBr) v: 3452, 1634, 1526, $1271 \mathrm{~cm}^{-1}$; MS m/z: $332[\mathrm{M}+\mathrm{H}]^{+}$. Anal. calcd for $\mathrm{C}_{18} \mathrm{H}_{18} \mathrm{ClNO}_{3} \mathrm{C} 65.16, \mathrm{H} \mathrm{5.47,} \mathrm{N} 4.22$; found $\mathrm{C}$ 65.48, H 5.65, N 4.40.
辅助材料(Supporting Information) 产物的核磁共振 氢谱和碳谱图. 这些材料可以免费从本刊网站(http:// sioc-journal.cn/)上下载.

\section{References}

[1] Zhou, W. J.; Zhang, L.; Xiao, W.; Chen, H. J.; Wu, W. N.; Ouyang, G. P. J. Heterocycl. Chem. 2017, 54, 1423.

[2] Praveen, C.; Nandakumar, A.; Dheenkumar, P.; Muralidharan, D.; P. Perumal, P. T. J. Chem. Sci. 2012, 124, 609.

[3] Temiz-Arpaci, O.; Arisoy, M.; Sac, D.; Doganc, F.; Tasci, M.; Senol, F. S.; Orhan, I. E. Z. Naturforsch., C 2016, 71, 409.

[4] Reen, G. K,; Kumar, A.; Sharma, P. Med. Chem. Res. 2017, 26 , 3336.

[5] Yildiz-Oren, I.; Yalcin, I.; Aki-Sener, E.; Ucarturk, N. Eur. J. Med. Chem. 2004, 9, 291.

[6] Tasci, M.; Temiz-Arpaci, O.; Kaynak-Onurdag, F.; Okten, S. Indian J. Chem., Sect. B: Org. Chem. Incl. Med. Chem. 2018, 57, 385.

[7] Tseng, C.-H.; Lin, C.-K.; Chen, Y.-L.; Tseng, C.-K.; Lee, J.-Y Lee, J.-C. Eur. J. Med. Chem. 2018, 143, 970.

[8] Henderson, J. A.; Bilimoria, D.; Bubenik, M.; Cadilhac, C.; Cottrell, K. M.; Denis, F.; Dietrich, E.; Ewing, N.; Falardeau, G. Giroux, S.; L'Heureux, L.; Liu, B.; Mani, N.; Morris, M.; Nicolas, O.; Pereira, O. Z.; Poisson, C.; Reddy, T. J.; Selliah, S.; Shawgo, R. S.; Vaillancourt, L.; Wang, J.; Xu, J.; Chauret, N.; Berlioz-Seux, F.; Chan, L. C.; Das, S. K.; Grillot, A.-L.; Bennani, Y. L.; Maxwell, J. P. Bioorg. Med. Chem. Lett. 2015, 25, 948.

[9] Zilifdar, F.; Foto, E.; Ertan-Bolelli, T.; Aki-Yalcin, E.; Yalcin, I.; Diril, N. Arch. Pharm. 2018, 351

[10] Khajondetchairit, P.; Phuangsawai, O.; Suphakun, P.; Rattanabunyong, S.; Choowongkomon, K.; Gleeson, M. P. Chem. Biol. Drug Des. 2017, 90, 987

[11] Goekhan-Kelekci, N.; Koeksal, M.; Uenuevar, S.; Aktay, G. Erdogan, H. J. Enzyme Inhib. Med. Chem. 2009, 24, 29.

[12] Eren, G.; Unlu, S.; Nunez, M. T.; Labeaga, L.; Ledo, F.; Entrena, A.; Banoglu, E.; Costantino, G.; Sahin, M. F. Bioorg. Med. Chem. 2010, 18, 6367.

[13] Jayanna, N. D.; Vagdevi, H. M.; Dharshan, J. C.; Raghavendra, R. Telkar, S. B. Med. Chem. Res. 2013, 22, 5814.

[14] Wei, P.-F.; Qi, M.-Z.; Wang, Z.-P.; Ding, S.-Y.; Yu, W.; Liu, Q.; Wang, L.-K.; Wang, H.-Z.; An, W.-K.; Wang, W. J. Am. Chem. Soc. 2018, 140, 4623.

[15] Yeh, V. S. C. Tetrahedron 2004, 60, 11995.

[16] Leaver, I. H.; Milligan, B. Dyes Pigm. 1984, 5, 109.

[17] Chen, T.-R. J. Organomet. Chem. 2008, 693, 3117.

[18] Dunwell, D. W.; Evans, D. Hicks, T. A. J. Med. Chem. 1975, 18, 1158.

[19] Grossi, G.; Di Braccio, M.; Roma, G.; Ballabeni, V.; Tognolini, M.; Calcina, F.; Barocelli, E. Eur. J. Med. Chem. 2002, 37, 933

[20] Smith, R. H., Jr.; Jorgensen, W. L.; Tirado-Rives, J. Lamb, M. L.; Janssen, P. A.; Michejda, C. J.; Kroeger Smith, M. B. J. Med. Chem. 1998, 41, 5272

[21] Liu, D.; Chen, H. Y.; Zhang, J. Y.; Huang, J. Y.; Li, Y. M.; Peng, Q. M. Appl. Surf. Sci. 2018, 456,59 .

[22] Li, X.-Q. Li; Wang, L.-Z. Chin. Chem. Lett. 2014, 25, 327.

[23] Yin, L.-Y.; Wang, L.-Z. Tetrahedron Lett. 2016, 57, 5935.

[24] Qiu, Z.-L.; Wang, L.-Z.; Li, W.-H.; Li. Y. Acta Chim. Sinica 2011, 69, 1217 (in Chinese).

(邱召来，王兰芝，李文红，李媛，化学学报, 2011, 69, 1217.)

(Li, L.; Fan, Y.) 